\title{
High aspect ratio nanotubes assembled from macrocyclic iminium salts
}

\author{
Chao Sun (孙超) ${ }^{a, b}$, Meng Shenc, Anton D. Chavez ${ }^{a, b}$, Austin M. Evans ${ }^{a}$, Xiaolong Liu ${ }^{d}$ ，Boris Harutyunyan ${ }^{\mathrm{e}}$ ， \\ Nathan C. Flanders ${ }^{\mathrm{a}}$, Mark C. Hersam ${ }^{\mathrm{a}, \mathrm{c}, \mathrm{d}}$, Michael J. Bedzyk ${ }^{\mathrm{c}, \mathrm{d}, \mathrm{e}}$, Monica Olvera de la Cruz ${ }^{\mathrm{a}, \mathrm{c}, \mathrm{d}, \mathrm{e}, 1}$, \\ and William R. Dichtel ${ }^{\mathrm{a}, 1}$
} aDepartment of Chemistry, Northwestern University, Evanston, IL 60208 ; ${ }^{\mathrm{b}}$ Department of Chemistry and Chemical Biology, Cornell University, Ithaca, NY
14853; 'Department of Materials Science and Engineering, Northwestern University, Evanston, IL 60208; ${ }^{\mathrm{d} A p p l i e d}$ Physics Graduate Program, Northwestern
University, Evanston, IL 60208; and 'Department of Physics and Astronomy, Northwestern University, Evanston, IL 60208

Contributed by Monica Olvera de la Cruz, July 20, 2018 (sent for review May 31, 2018; reviewed by Jonathan L. Sessler and Gregory A. Voth)

\begin{abstract}
One-dimensional nanostructures such as carbon nanotubes and actin filaments rely on strong and directional interactions to stabilize their high aspect ratio shapes. This requirement has precluded making isolated, long, thin organic nanotubes by stacking molecular macrocycles, as their noncovalent stacking interactions are generally too weak. Here we report high aspect ratio $\left(>10^{3}\right)$, lyotropic nanotubes of stacked, macrocyclic, iminium salts, which are formed by protonation of the corresponding imine-linked macrocycles. Iminium ion formation establishes cohesive interactions that, in organic solvent (tetrahydrofuran), are two orders of magnitude stronger than the neutral macrocycles, as explained by physical arguments and demonstrated by molecular dynamics simulations. Nanotube formation stabilizes the iminium ions, which otherwise rapidly hydrolyze, and is reversed and restored upon addition of bases and acids. Acids generated by irradiating a photoacid generator or sonicating chlorinated solvents also induced nanotube assembly, allowing these nanostructures to be coupled to diverse stimuli, and, once assembled, they can be fixed permanently by cross-linking their pendant alkenes. As large macrocyclic chromonic liquid crystals, these iminium salts are easily accessible through a modular design and provide a means to rationally synthesize structures that mimic the morphology and rheology of carbon nanotubes and biological tubules.
\end{abstract}

stimuli-responsive materials | macrocycles | supramolecular chemistry | nanotubes | molecular dynamics

$\mathbf{N}$ anotubes offer unique properties that emerge from their low dimensionality, tubular rigidity, and confined interior microenvironments (1). These features have been exploited for the directional or selective transport of charges $(2,3)$, energy (4), biomass (5), and water (6); for the mechanical reinforcement of living cells (5) and soft matter (7); and as containers for drugs and viral genetic information (8). Their few-nanometer thickness and high aspect ratios impart these functions and are key to their rheological properties and processability. Inspired by biological 1D nanostructures such as microtubules and helical virus capsids, abiotic self-assembled nanotubes have been explored based on cylindrical micelles (9) and columns using helical (3) or tapered (10) building blocks. These designs sometimes provide micrometer-long structures (9), but with thicknesses that are 10 times that of carbon nanotubes (CNTs). Aida and coworkers (9) noted that cylindrical micelles derived from amphiphile bilayers were approaching their ultimate thickness limits. Molecular macrocycles (MCs) are intuitive building blocks for thinner nanotubes because their composition, size, and porosity can be defined rationally through chemical synthesis (11). However, MC stacking is generally too weak to produce nanotubes with the high aspect ratios $\left(>10^{3}\right)$ of CNTs and biological filaments. Stacked MCs instead almost always exhibit aspect ratios around 10 as short, individual assemblies or longer structures stabilized by bundling (12-14), limiting their utility as isolated nanostructures (15). Alternatively, when functionalized with many long alkyl or alkyloxy side chains, they form bulk liquid crystalline phases
(16-19), with assembly sometimes driven by the presence of metal ions or organic guests (20). Shionoya and coworkers (21) recently observed that cyclometallated $\mathrm{Pd}$-phenanthroline MCs formed micrometer-long nanotubes that were attributed to the squareplanar geometry of the metal; the corresponding $\mathrm{Zn}$ complexes formed spherical aggregates. However, a general strategy has yet to emerge for making synthetic nanotubes that rival the morphologies of isolated CNTs.

Here we describe MCs that stack into lyotropic nanotubes with aspect ratios $>10^{3}$ upon protonation of their imine linkages, which turns on cohesive interactions of $10^{2}$ to $10^{3} \mathrm{~kJ} / \mathrm{mol}$ as estimated by molecular dynamics simulations. Stacking stabilizes the MC's 12 iminium linkages, which are normally prone to hydrolysis, and is reversed and restored through the addition of bases and acids, respectively, including acids introduced by irradiating a photoacid generator or sonicating chlorinated solvents. Reactive alkene groups on the periphery of the MCs were cross-linked using thiol-ene reactions, thereby stabilizing the nanotubes as covalently bonded structures. These macrocyclic iminium salts feature a modular design via one-step synthesis from readily accessible amine and aldehyde components. As a diverse family of large ( $>3,500$ daltons) and stimuli-responsive chromonic liquid crystals $(20,22)$, they provide a means to rationally access and manipulate high aspect ratio nanotubes with modular designs. Accessing high aspect ratio nanotubes based on

\section{Significance}

The distinct properties of one-dimensional nanomaterials, such as carbon nanotubes and actin filaments, emerge from their high aspect ratios. Stacking macrocycles into nanotubes represents a potentially powerful design strategy to access synthetic nanotubes, but such noncovalent assemblies are typically too weak to support individual, high aspect ratio structures. We report hexagonal macrocycles that exhibit strong electrostatic stacking upon protonation of their imine linkages. The resulting nanotubes form lyotropic phases and align in flow, a hallmark of high aspect ratio structures. The iminium-linked macrocycle assemblies form and disassemble reversibly in the presence of bases and acids, and are stabilized by covalent cross-linking.

Author contributions: C.S., M.S., A.D.C., M.O.d.I.C., and W.R.D. designed research; C.S., M.S., A.D.C., A.M.E., X.L., N.C.F., and M.C.H. performed research; C.S., M.S., A.D.C., B.H., M.J.B., M.O.d.I.C., and W.R.D. analyzed data; and C.S., M.S., A.D.C., M.O.d.I.C., and W.R.D. wrote the paper.

Reviewers: J.L.S., University of Texas at Austin; and G.A.V., The University of Chicago. The authors declare no conflict of interest.

Published under the PNAS license.

${ }^{1}$ To whom correspondence may be addressed. Email: m-olvera@northwestern.edu or wdichtel@northwestern.edu.

This article contains supporting information online at www.pnas.org/lookup/suppl/doi:10. 1073/pnas.1809383115/-/DCSupplemental.

Published online August 21, 2018.

(1)


designed molecular assemblies represents a key challenge for making stimuli-responsive nanostructures with rheological and structural properties approaching biological systems and CNTs. Such nanotubes could serve as dynamic cytoskeletons in artificial protocells (23), backbones of hybrid nanomachines inspired by complex biological machineries such as bacterial contractile ejection systems $(24,25)$, organizing elements of memory $(26)$ or electrical energy storage devices (27-29), and other applications that emerge from lyotropic liquid crystals $(7,30,31)$.

\section{Results and Discussion}

A hexagonal MC (1-DMTP, Fig. 1A) containing 12 imine linkages forms in a single step in $52 \%$ isolated yield by condensing equimolar amounts of an alkene-terminated triphenylbenzene diamine (1) (SI Appendix, Scheme S1) and dimethoxyterephthaldehyde (DMTP) (SI Appendix, Scheme S1). The MCs exhibit a single narrow peak in gel permeation chromatography (GPC) (SI Appendix, Fig. S1) as well as the expected peak at $m / z=3556.8\left([\mathrm{M}+\mathrm{H}]^{+}\right)$ by matrix-assisted laser desorption/ionization mass spectrometry (MALDI-MS) (SI Appendix, Fig. S2). The alkene functional groups of 1-DMTP were chosen for subsequent cross-linking (see below), and the assembly of this species is described in detail below. Here we also emphasize the modularity of this design strategy by synthesizing three other MCs: one with decyloxy side chains (2) (SI Appendix, Scheme S2), another using terephthaldehyde (SI Appendix, Scheme S3), and the third using an anthracene-containing

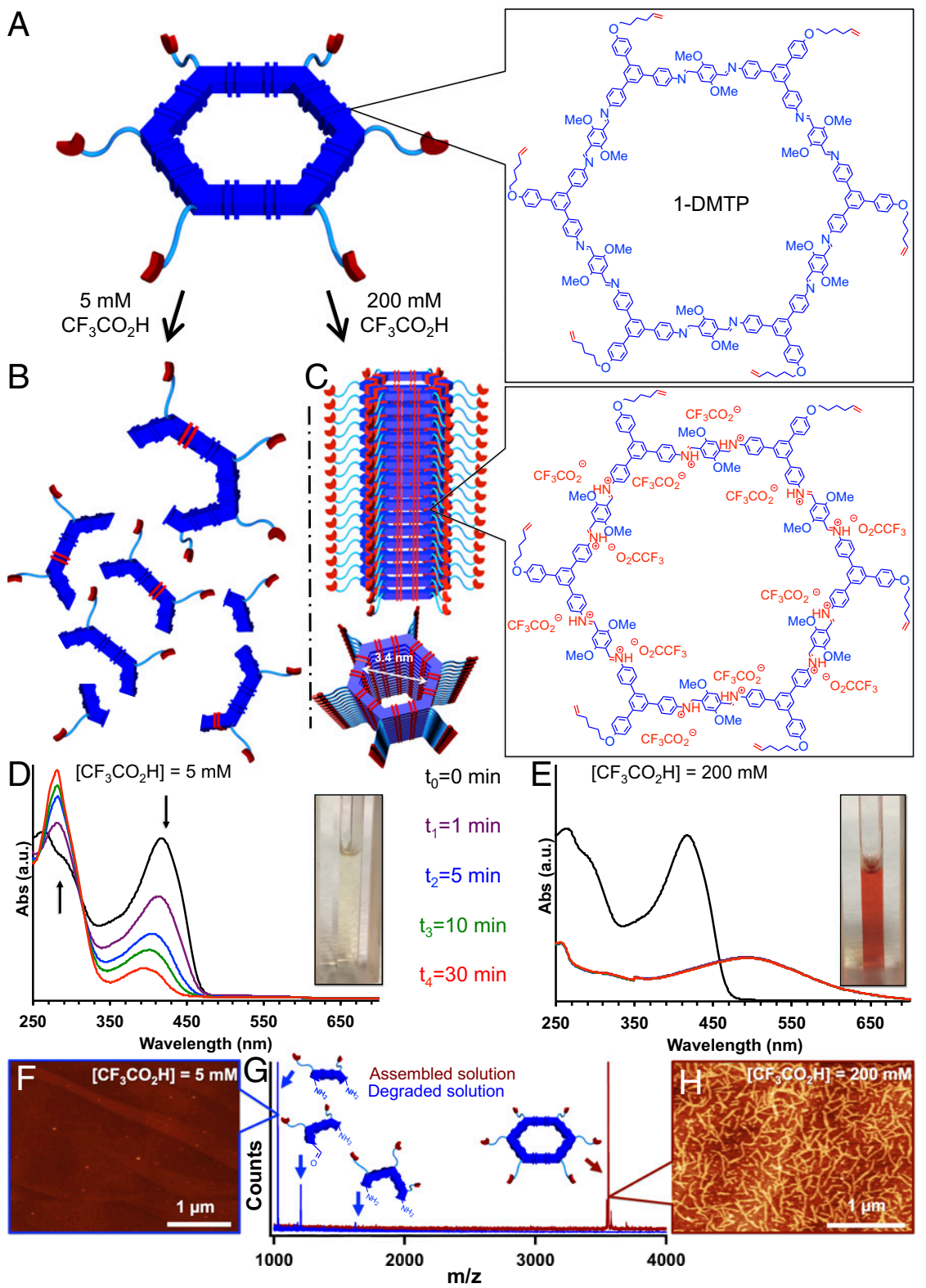

Fig. 1. Imine-linked MCs assemble at high acid concentration but degrade at low acid concentration. (A) Structure of a hexagonal MC 1-DMTP containing 12 imine linkages. The MCs were dissolved in THF $\left(100 \mu \mathrm{M}\right.$; with $3 \mathrm{mM}$ TBAP) in the presence of $\mathrm{CF}_{3} \mathrm{CO}_{2} \mathrm{H}$ at low $(5 \mathrm{mM})$ and high $(200 \mathrm{mM})$ concentrations. $(B)$ The lower $\mathrm{CF}_{3} \mathrm{CO}_{2} \mathrm{H}$ concentration $(5 \mathrm{mM})$ is insufficient to induce $\mathrm{MC}$ stacking and instead catalyzes degradation into smaller fragments. $(C)$ The higher $\mathrm{CF}_{3} \mathrm{CO}_{2} \mathrm{H}$ concentration $(200 \mathrm{mM})$ protonates most or all of the imines, and the resulting iminium-containing MCs assemble into nanotubes. (D and $E$ ) UV-vis spectra as a function of time after introducing $(D) 5 \mathrm{mM} \mathrm{CF}_{3} \mathrm{CO}_{2} \mathrm{H}$ or $(E) 200 \mathrm{mM} \mathrm{CF}_{3} \mathrm{CO}_{2} \mathrm{H}$ (Insets, photograph at $t=30 \mathrm{~min}$ ). (F) AFM of the $5 \mathrm{mM} \mathrm{CF}_{3} \mathrm{CO}_{2} \mathrm{H}$ solution drop-cast on HOPG. (G) MALDI-MS of

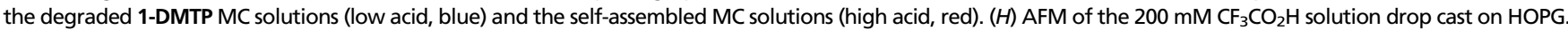


dialdehyde (SI Appendix, Scheme S4). Each of these MCs is dispersible up to $100 \mu \mathrm{M}$ in THF with no evidence of assembly and precipitates as a microcrystalline powder from polar solvents ( $S I$ Appendix, Fig. S17) (32).

The 1-DMTP MCs exhibit a counterintuitive stability as a function of acid concentration that reflects both their acid-labile imine linkages and protonation-induced assembly. THF solutions of 1-DMTP $(100 \mu \mathrm{M})$ containing $5 \mathrm{mM} \mathrm{CF}_{3} \mathrm{CO}_{2} \mathrm{H}$ undergo rapid hydrolysis to $\mathrm{MC}$ fragments within minutes (Fig. $1 B$ ), as evidenced by a change in solution color (from yellow to colorless) and evolution of its UV-visible (UV-vis) spectrum over $30 \mathrm{~min}$ (Fig. $1 D$ ) (33). After this change, further addition of TFA up to $200 \mathrm{mM}$ does not alter the UV-vis spectrum significantly (Fig. 1D, Inset). MALDI-MS of these degraded solutions exhibits peaks at $\mathrm{m} / \mathrm{z}$ of $1,027(2 \cdot \mathbf{1}+$ DMTP $), 1,204(2 \cdot \mathbf{1}+2 \cdot$ DMTP $)$, and 1,620 (3 $\cdot \mathbf{1}+$ $2 \cdot$ DMTP), all of which correspond to MC fragments (Fig. $1 G$ ). In contrast, an otherwise identical THF solution of 1-DMTP containing more $\mathrm{CF}_{3} \mathrm{CO}_{2} \mathrm{H}(200 \mathrm{mM})$ and tetrabutylammonium perchlorate (TBAP; $3 \mathrm{mM}$ ) immediately turns red-orange (Fig. $1 E$, Inset), corresponding to a red shift in absorption from $416 \mathrm{~nm}$ to $493 \mathrm{~nm}$ (Fig. $1 E$ ), which is characteristic of conjugated iminium ions (33). Infrared spectroscopy of 1-DMTP was also consistent with iminium ion formation, as the characteristic $\mathrm{C}=\mathrm{N}$ stretches were blue-shifted and broadened (SI Appendix, Fig. S23) (34). MALDI-MS analysis of the solution provided a single prominent peak at $m / z=3,556.8$, corresponding to $[\mathrm{M}+\mathrm{H}]^{+}$of the intact $\mathrm{MC}$. The presence of $3 \mathrm{mM}$ TBAP into MC solutions at $5 \mathrm{mM}$ TFA does not affect the UV-vis evolution in Fig. $1 D$, but was used to be consistent with imaging experiments described below. Together, these data indicate that 1-DMTP is unusually stable in the presence of $200 \mathrm{mM} \mathrm{CF}_{3} \mathrm{CO}_{2} \mathrm{H}$ yet unstable at $5 \mathrm{mM} \mathrm{CF}_{3} \mathrm{CO}_{2} \mathrm{H}$.

The unexpectedly high stability of 1-DMTP to high (but not low) concentrations of $\mathrm{CF}_{3} \mathrm{CO}_{2} \mathrm{H}$ is associated with $\mathrm{MC}$ assembly. Atomic force microscopy (AFM) of solutions of 1-DMTP containing $200 \mathrm{mM}$ $\mathrm{CF}_{3} \mathrm{CO}_{2} \mathrm{H}$ drop cast on highly ordered pyrolytic graphite (HOPG) indicated 1D assemblies with uniform widths and 4-nm thicknesses (Fig. $1 H$ and SI Appendix, Fig. S19). No features were observed in micrographs of drop cast solutions of 1-DMTP containing $5 \mathrm{mM}$ $\mathrm{CF}_{3} \mathrm{CO}_{2} \mathrm{H}$ (Fig. $1 F$ ). The role of $\mathrm{MC}$ stacking in stabilizing iminium linkages at high acidity is further supported by a model compound containing two imine linkages (SI Appendix, Scheme S8 and Fig. S24), which hydrolyzes in the presence of $200 \mathrm{mM} \mathrm{CF}_{3} \mathrm{CO}_{2} \mathrm{H}$. All of the imine-linked MCs described above assemble similarly under high $\mathrm{CF}_{3} \mathrm{CO}_{2} \mathrm{H}$ concentrations (SI Appendix, Schemes S2-S4 and Fig. S19). The 1-DMTP MC is studied in greatest detail below because it is capable of covalent cross-linking, but these findings indicate a general design for nanotubes that is based on directional bonding approaches and relatively simple monomers. The $\mathrm{MC}$ assemblies continue to evolve over $12 \mathrm{~h}$, after which flocculation of red fibrous solid leaves a nearly colorless solution (SI Appendix, Fig. S18), indicating the depletion of dissolved MCs. AFM further reveals that the red solutions containing short tubular assemblies (Fig. $1 H$ ) ripen into micrometer-long nanotubes with aspect ratios exceeding 1,000, which entangle into meshes (Fig. $2 A$ ). Even in these relatively dense meshes, bundles of nanotubes are rare (Fig. $2 A$ ). We attribute the formation of individual nanostructures, in part, to the TBAP electrolyte, whose ions screen side-by-side interactions between adjacent nanotubes (Fig. $2 B$ ). Micrographs obtained of nanotubes in the presence of smaller cations or anions $\left[\mathrm{LiClO}_{4}\right.$, $\mathrm{NBu}_{4} \mathrm{Br}, \mathrm{N}\left(\mathrm{CH}_{3}\right)_{4} \mathrm{ClO}_{4}$ ] showed more prevalent bundled structures, whereas another large, noncoordinating electrolyte pair $\left(\mathrm{NBu}_{4} \mathrm{PF}_{6}\right)$ also provided mostly separated nanotubes (SI Appendix, Fig. S20). Overall, the few-nanometer diameter and thickness of nanotubes under AFM are consistent with the size of MCs (3.4-nm inner diameter). The nanotubes extend for several microns with 5-nm diameters in solution, as characterized using transmission electron microscopy (TEM) and polarized light imaging (Fig. $2 C$ and $D$ ). The transmission electron micrographs indicate a uniform tube

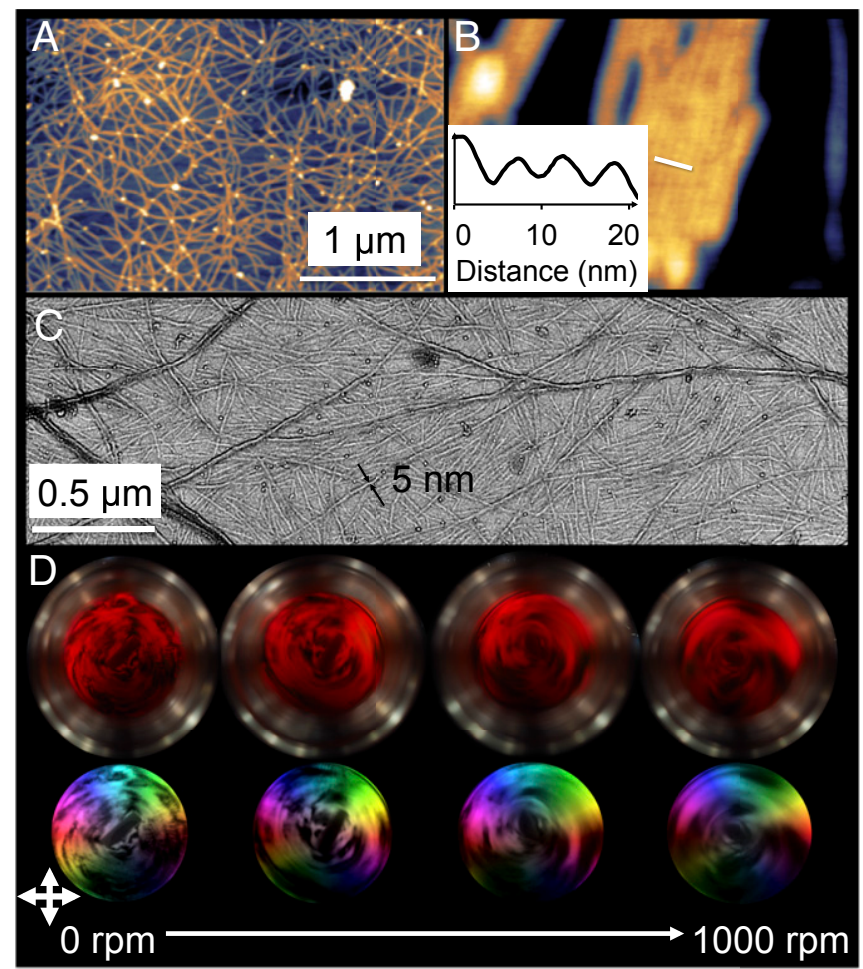

Fig. 2. Morphology and LC behavior of the nanotubes. (A) AFM of a mesh of nanotubes from $100 \mu \mathrm{M}$ 1-DMTP solution containing $200 \mathrm{mM}$ TFA and $3 \mathrm{mM}$ TBAP drop cast on HOPG. (B) AFM of a rare bundle of nanotubes arranged on HOPG indicates an approximate 5-nm width of individual nanotubes (Inset). (C) TEM of dense, nonbundled nanotubes with uniform, 5-nm widths and micrometerscale lengths. $(D)$ The lyotropic nanotubes shown align in the circular flow generated by stirring the suspension. (Top) True colors of the LC phases photographed through cross-polarizers at various stirring rates. (Bottom) False-colored photographs in which the color gradient represents the orientation of the LC phases.

diameter consistent with the sizes of individual 1-DMTP MCs. The nanotubes appear rigid with micrometer-long persistent lengths and morphologies comparable to multiwalled CNTs. Tube ends are rarely observed, suggesting high end-cap energies. Although we observe weak electron diffraction spots from the nanotubes dispersed onto a TEM grid, the nanotubes do not appear to be 1D crystals (SI Appendix, Fig. S21).

Grazing incidence wide-angle X-ray scattering of a drop-cast film indicated a stacking distance of $4.6 \AA$ between MCs with a preferential orientation normal to the HOPG substrate (SI Appendix, Fig. S22 $A$ and $B$ ). A polarized light micrograph reveals the spontaneously ordered domains (SI Appendix, Fig. S22E). Concentrated nanotube suspensions exhibit a packing distance of $56.9 \AA$ (SI Appendix, Fig. S22 $C-F$ ) via transmission X-ray diffraction, characteristic of the $\mathrm{M}$ phases in chromonic liquid crystals (22); this signal is absent in dilute nanotube suspensions. Because of their high aspect ratios (35), these nanotubes exhibit liquid crystalline (LC) behavior in solution at concentrations as low as $0.04 \mathrm{wt} \%$ of the MC (Fig. $2 D$ ). Similar behavior has been reported for dispersions of multiwalled CNTs (30). The LC phases respond to flow generated by stirring in a glass vial (Fig. $2 D$ ). Increasing flow rate induces greater nanotube alignment, as indicated by increasingly homogeneous red-colored phases (Fig. 2D, Top) and less segregated phase orientations (Fig. 2D, Bottom; see also Movie S1). Both of these image sets show increased continuity at higher stirring rate, demonstrating excellent shear alignment properties of nanotubes with circular flow. These results indicate that intriguing rheological behaviors emerge with low concentrations of high aspect ratio nanotubes assembled from macrocyclic iminium salts for nanotube alignment and processing. 
The rapid and robust assembly of cationic iminium-linked MCs is superficially unexpected, considering the apparent presence of charge repulsion. However, in low-dielectric constant media such as THF, small amounts of salts can significantly alter phase behavior (36) because correlations among charges are very strong $(37,38)$. We theorize that, upon protonation, cationic $\mathrm{MCs}$ attract $\mathrm{CF}_{3} \mathrm{CO}_{2}{ }^{-}$ counterions and aggregate into nanotubes containing alternating structures of cationic $\mathrm{MCs}$ and $\mathrm{CF}_{3} \mathrm{CO}_{2}^{-}$anions via electrostatic interactions. Acids with different counterions such as $\mathrm{HCl}$ also induce MC stacking (see below). The assembly process and the role of the counterions were investigated using molecular dynamics simulations by explicitly including the unprotonated and cationic 1-DMTP MCs, the THF solvent molecules, and $\mathrm{CF}_{3} \mathrm{CO}_{2}{ }^{-}$counterions. Two unprotonated MCs placed $20 \AA$ apart (Fig. $3 A$, Inset) remain separated during a 30 -ns simulation (Fig. $3 A$ and Movie S2), while protonated MCs initially separated by $20 \AA$ (Fig. 3B, Inset) aggregate within $1 \mathrm{~ns}$ of the simulation with four pairs of protonated sites bound together with counterions for the entire 50-ns simulation (Fig. $3 B$ and Movie S2). Furthermore, two preassembled nonprotonated
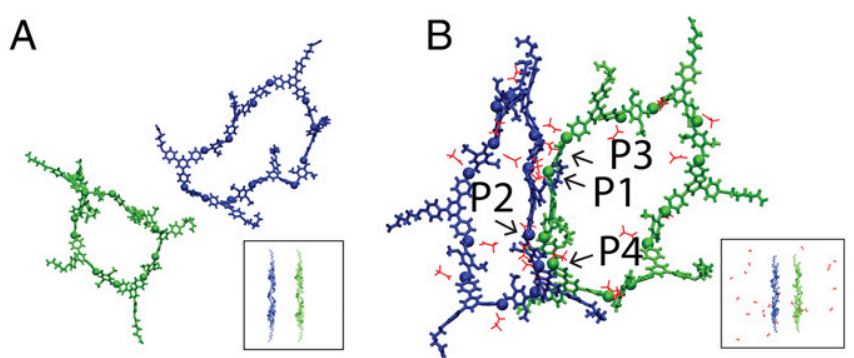

C

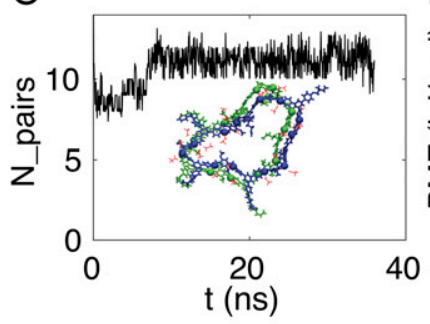

$\mathrm{D}$

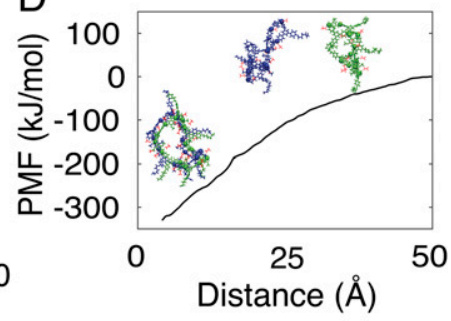

E

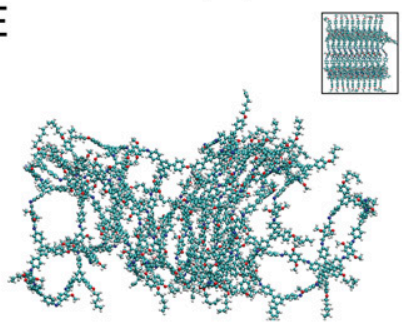

F

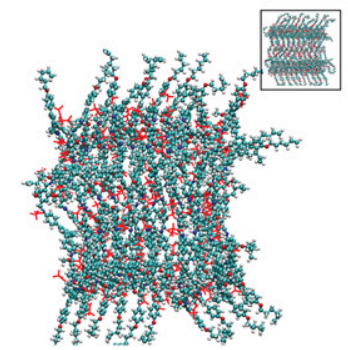

Fig. 3. Molecular dynamics simulations of acid-mediated stacking. ( $A$ and $B$ ) Representative depictions of $(A)$ two neutral and $(B)$ two protonated 1-DMTP MCs that were initially separated during the simulations (Insets). The spheres represent the imine and iminium $\mathrm{N}$ atoms in $A$ and $B$, respectively. Red structures in $B$ represent $\mathrm{CF}_{3} \mathrm{CO}_{2}^{-}$counterions. The THF solvent molecules included in the simulations are excluded for clarity. Arrows P1 to P4 in B point to four protonated $\mathrm{N}-\mathrm{N}$ pairs that are bound across the two MCs through counterions. $(C)$ The number of bound $\mathrm{N}-\mathrm{N}$ pairs between the two preassembled protonated MCs. (Inset) A snapshot of the simulation. $(D)$ The PMF from umbrella sampling along the collective variable of the distance between the center of mass of Ns of two preassembled protonated MCs. The error bars of the PMF, estimated by Monte Carlo bootstrap error analysis, are within $1 \mathrm{~kJ} \cdot \mathrm{mol}^{-1}$. (Inset) The snapshots for the first (assembled) and the last (dissembled) umbrella sampling windows. ( $E$ and $F$ ) Representative snapshots of $10(E)$ preassembled MCs and $(F)$ fully protonated MCs during 36-ns simulation. (Insets) The prearranged 10-ring stacks at the beginning of the simulation.
MCs become fully separated after 24 ns (Movie S3), with insignificant cohesive energy that is reproduced in the case of 10 preassembled MCs (SI Appendix, Fig. S28A), while protonated MCs stay assembled (Fig. 3C, Inset and Movie S3). The number of bound $\mathrm{N}-\mathrm{N}$ pairs between the two protonated MCs remains $\sim 12$ (Fig. $3 C$ ).

Simulations reveal a strong cohesive energy between protonated MCs. In a direct calculation without biases, the trajectories of 10 preassembled protonated MCs show strong cohesive energy per MC ( - 1,310 $\mathrm{kJ} \cdot \mathrm{mol}^{-1}$, i.e., $\sim 530 k_{B} T$, where $k_{B} T$ is the thermal energy), with the electrostatic energy being the major contribution (SI Appendix, Fig. S28A). SI Appendix, Fig. S28B shows that the magnitude of $\Delta \mathrm{E}_{\mathrm{vdw}}$, the difference of the van der Waals interaction energy per macrocycle between the assembled and the dissembled states, for both the protonated and the deprotonated MCs is more than 10 times smaller than the electrostatic interaction for the protonated MCs, which corroborates that the self-assembly of protonated MCs is driven by the electrostatic attraction. The potential of mean force (PMF) using umbrella sampling (39) also indicates a strong attraction of $\sim 350 \mathrm{~kJ} \cdot \mathrm{mol}^{-1}$, i.e., $\sim 140 k_{B} T$, between the two protonated MCs (Fig. $3 D$; see also Movie S4). The PMF difference from umbrella sampling is much smaller than that from direct interaction energy calculation for two reasons: First, the PMF includes both entropic and enthalpic contributions, which counteract each other. Second, the direct energy estimate is for at least $10 \mathrm{MCs}$, while the umbrella sampling is performed for two MCs, which neglects longer-range effects. We note that sampling for the self-assembly of the MCs is challenging due to many degrees of freedom, including the distance, the tilting angle, the ring conformation, and the counterion coordination. An accurate analysis would require advanced methods for finding the true reaction pathway $(40,41)$. Nevertheless, the PMF difference from umbrella sampling here has the same sign and is in the same order of magnitude as the direct interaction energy calculation, indicating that electrostatic interaction dominates the self-assembly process.

We also estimate, from a simple electrostatic calculation, the cohesive energy $(E)$ of an electroneutral unit in the nanotube aggregate (where the 12 charges per MC are neutralized by counterions) that considers only the first nearest neighbors of the protonated sites and the counterions: $E / k_{B} T=12\left(-l_{B} / r_{H N-C I}\right)$ where $l_{B}=7.3 \mathrm{~nm}$ is the Bjerrum length in THF $\left(l_{B}=e^{2} / 4 \pi \varepsilon_{0} \varepsilon_{r} k_{B} T\right.$, where $\varepsilon_{r}$ is the relative permittivity and $e$ is the unit charge) and $r_{H N-C I}=0.295 \mathrm{~nm}$ is the peak distance of the $\mathrm{HN}-\mathrm{CI}$ radial distribution, where $\mathrm{HN}$ denotes the proton attached to $\mathrm{N}$ and $\mathrm{CI}$ denotes the counterions. The interaction energy, $E\left(-297 k_{B} T\right.$, i.e., $\left.-740 \mathrm{~kJ} \cdot \mathrm{mol}^{-1}\right)$, is on the same order of magnitude as the interaction calculated from the direct interaction energy calculation. This calculation underestimates $E$ because it excludes higher-order contributions of alternating sign from the positive and negative charges located at longer distances, which, in periodic systems, can be resumed to add a multiplicative factor of order one (the Madelung constant) to $E$ and, in disordered systems, also gives minor corrections (42). Furthermore, the 10-ring assembly of neutral MCs (Fig. $3 F$, Inset) falls apart quickly during the 36-ns simulations (Fig. $3 E$ and Movie S5), while that of protonated MCs (Fig. 3E, Inset) remains aggregated throughout (Fig. $3 F$ and Movie S6). The simulated stacking distance between MCs (SI Appendix, Fig. S29) is consistent with the experimental value measured by X-ray diffraction (SI Appendix, Fig. S22A). The simulations also suggest that the interior of the nanotube accommodates $\sim 300$ disordered THF molecules for every 10 stacked rings.

Similar to actin filaments and CNTs, which sustain their high aspect ratios from fracture with strong, directional protein interfaces and covalent bonds $\left(10^{2} \mathrm{~kJ} / \mathrm{mol}\right)(43)$, we note that electrostatic cohesion is comparably strong. In low-dielectric constant media, excess charge is strongly penalized and ionic correlations, which are neglected in Poisson-Boltzmann approaches (44), are strong, resulting in the formation of neutral ion pairs (or cluster when geometrically possible) that strongly decreases the free energy of the systems (45-47); for comparison, the energy to pull 
apart two monovalent charges of opposite charge touching at $\sim 0.3 \mathrm{~nm}$ in THF is $\sim 60 \mathrm{~kJ} / \mathrm{mol}$. This electrostatic driven short range interaction, which for MCs with 12 charges leads to robust electroneutral aggregates (Fig. $3 F$ ), is stronger than van der Waals interactions and highly orientation-dependent hydrogen bonds (each $10^{0}$ to $10^{1} \mathrm{~kJ} / \mathrm{mol}$ ) (48). The combined stacking characteristics enable the formation of isolated nanotubes with high aspect ratios. Stacking interactions of this strength are unavailable to other layered assemblies of MCs, such as hydrogen-bonded cyclic peptides, which do not achieve micrometer lengths without forming crystalline bundles of low aspect ratios (15). Long-ranged, orientation-insensitive interactions tolerate some longitudinal disorder (Fig. $3 F$ ), consistent with the only weak electron diffraction observed when evaluating the 1D crystallinity of the nanotubes (SI Appendix, Fig. S21B). Nevertheless, the simulations show MCs stacked with nitrogens in different layers close to each other, in a near-eclipsed way (Fig. $3 F$; see also SI Appendix, Fig. S26D) such that these nanotubes exhibit uniform widths over micrometer lengths (SI Appendix, Fig. S21D) and interiors for solvents, metal ions (SI Appendix, Fig. S21E), or other future payloads. These results show the importance of multivalent electrostatic interactions in sustaining the cohesive interface and preserving MC integrity.

By alternating between acidic and basic conditions, the 1-DMTP $\mathrm{MC}$ solutions switch between a neutral, imine-linked state that have cohesion energy of only a few times of the thermal energy $\left(k_{B} T\right)$ and an iminium-linked state with hundreds of $k_{B} T$ cohesive interactions, which assemble sufficiently rapidly to bypass MC degradation (see also Movie S7). A strong LC effect switches off and on correspondingly. We observe nucleation of short nanotubes immediately after $\mathrm{CF}_{3} \mathrm{CO}_{2} \mathrm{H}$ addition (Fig. $4 A$ ) and observe coalesced micrometerlong nanotubes after $12 \mathrm{~h}$ (Fig. $4 B$ ). The iminium nanotubes disassemble upon neutralization with $\mathrm{NEt}_{3}$ (Fig. $4 C$ ), and the solution

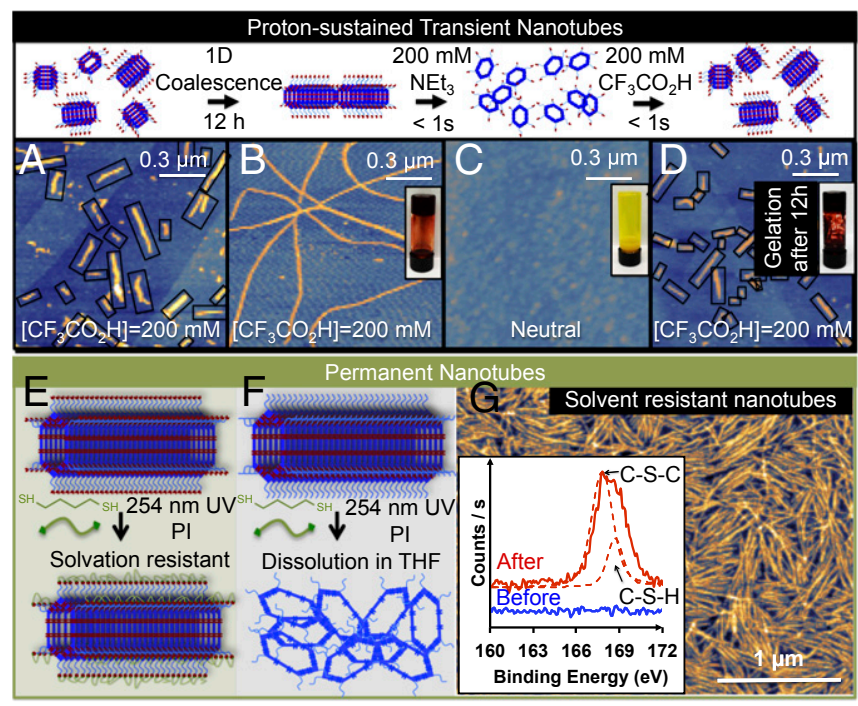

Fig. 4. Stimuli responsiveness of iminium nanotubes and their cross-linking. ( $A$ $D)$ AFM images and schematics of $(A)$ small nanotube segments (in black rectangular boxes) formed immediately after acidifying MC solutions; $(B)$ ripening to extended nanotubes, with (Inset) gelation of a concentrated nanotube solution; (C) disassembly of nanotubes upon neutralizing with $\mathrm{NEt}_{3}$, with (Inset) MC solution after NEt3 addition; and $(D)$ reformed short nanotubes (in black rectangular boxes) after adding $\mathrm{CF}_{3} \mathrm{CO}_{2} \mathrm{H}$, with (Inset) second gelation of the solution after ripening overnight. $(E)$ Cross-linking of the alkene side chains of the nanotubes using thiol-ene reactions, which provides solvent resistance of nanotubes. $(F)$ Nanotubes of imine-linked MC 2-DMTP lack alkenes and cannot be cross-linked (G) AFM image of solvation-resistant nanotubes cross-linked on HOPG. (Inset) XPS of S2p peaks before (blue) and after (red) the cross-linking reaction. returns to the yellow color of the neutral MCs (Fig. $4 C$, Inset). The nanotubes reform when more $\mathrm{CF}_{3} \mathrm{CO}_{2} \mathrm{H}$ is added (Fig. $4 D$ ). Concentrated MC solutions gel during ripening (Fig. $4 B$, Inset), flow after neutralization (Fig. $4 C$, Inset), and gel again upon replenishment of $\mathrm{CF}_{3} \mathrm{CO}_{2} \mathrm{H}$ and another ripening period (12 h; Fig. $4 D$ ). Acids sustain MC assembly in solutions similarly to how bound ATP molecules induce and sustain actin filaments in cells (5). Inspired by this process, two additional methods to stimulate nanotube formation are demonstrated in Movie S8, by either irradiating a photoacid generator or sonicating 1,2-dichloroethane to release $\mathrm{HCl}$ (see $\mathrm{SI}$ Appendix for detailed procedures) (49). Finally, the permanent cross-linking of nanotubes via their pendant alkenes was successful both on surface and in suspension using a photoinitiated thiol-ene click reaction, after which the nanotubes no longer disassemble by adding base (Fig. $4 E$ and SI Appendix, Fig. S25E), compared with alkene-absent MCs (Fig. 4F; see SI Appendix for detailed procedures). X-ray photoelectron spectroscopy (XPS) further indicates the primary presence of sulfur in $\mathrm{C}-\mathrm{S}-\mathrm{C}$ valence (Fig. $4 G$, Inset) (50). Electron micrographs show mostly isolated nanotubes after cross-linking (SI Appendix, Fig. S25 B and C). Intertubular crosslinking is likely unflavored, because the dithiol used is quite short relative to the large intertubular distances found even in concentrated suspensions (SI Appendix, Fig. S22). Control experiments on MCs lacking reactive alkene groups, omitting the 1,4-butanedithiol cross-linker or photoinitiator, or not irradiating the solution (SI Appendix, Fig. S25 D-G) all resulted in nanotubes that disassembled upon the addition of $\mathrm{NEt}_{3}$.

\section{Conclusions}

Hexagonal imine-linked MCs bearing alkyl solubilizing groups form high aspect ratio nanotube assemblies upon protonation, which stabilize their otherwise sensitive iminium ion linkages. The assemblies represent a diverse class of chromonic liquid crystals, of which the MCs are the largest discrete molecules and first iminiumcontaining structures known to assemble in this way. Molecular dynamics reveal strong, multivalent, electrostatic stacking that is comparable to carbon-carbon bonds as a strategy to assemble robust chromonic liquid crystals through aspect ratio control. The nanotubes exhibit emergent rheological properties such as acidresponsive gel formation and flow-responsive LC behavior with excellent shear alignment properties. This strategy provides access to isolatable, thin, long organic nanotubes with diverse structures through design of monomers and channel geometries such as square and pentagonal 1D space (11), opening up synthetic routes to functional nanotubes with accessible interiors for $1 \mathrm{D}$ transport and encapsulation, LC engineering (51), and abiotic matter capable of active transport. These nanotubes open up explorations into assembling $1 \mathrm{D}$ reactive salt stabilized by multivalent interactions, nuanced charge arrangement along 1D electrostatic structures, anion binding in MC assemblies $(52,53)$, reversible stacking mechanisms in layered 2D materials, and rheological studies of their micelle-like complex fluids unavailable to previous short, or bundled nanotubes.

\section{Materials and Methods}

For a complete set of detailed materials and methods, please see SI Appendix.

Reagents and Instrumentation. Reagents were purchased in reagent grade from commercial sources and used without further purification, unless otherwise described. Compounds S2 and 2 were synthesized as reported previously (SI Appendix). MC solutions of various concentrations were prepared by dissolving purified MC solids in appropriate solvents assisted by bath sonication. See SI Appendix for instrumentation details.

General Synthesis of MCs. Diamine (1 or 2 in SI Appendix) and dialdehyde (DMTP, TP, or AntTP, 1.0 equiv; see SI Appendix) were dissolved in 1,4-dioxane (20 mM each) before $\mathrm{CF}_{3} \mathrm{CO}_{2} \mathrm{H}$ ( 0.5 equiv) was added. A precipitate formed within minutes. The solutions were left to sit for $1 \mathrm{~d}$ to $6 \mathrm{~d}$. The acid was neutralized by the addition of $\mathrm{NEt}_{3}$ (10 equiv). The precipitate was isolated by 
centrifugation and subsequently rinsed with $\mathrm{Et}_{2} \mathrm{O}(15 \mathrm{~mL} \times 3)$. The solid was dried under vacuum at room temperature before being analyzed using GPC and MALDI-MS. See SI Appendix for more details of individual MCs.

Nanotube Sample Preparation for UV-Vis, AFM, TEM, X-Ray Diffractions, CrossPolarized Light Photography, and IR. An MC 1-DMTP solution was prepared in THF with $3 \mathrm{mM}$ TBAP. The solution was then subjected to $200 \mathrm{mM} \mathrm{CF}_{3} \mathrm{CO}_{2} \mathrm{H}$ for nanotube assembly. The nanotube solutions were used for UV-vis and cross-polarized light photography at appropriate concentrations. The dropcast samples of the solution were then used for AFM, TEM, and X-ray diffraction. The precipitates of the solution by centrifugation were used for IR. See SI Appendix for the details of individual characterizations.

Molecular Dynamics Simulations. MD simulations were performed using the package Nanoscale Molecular Dynamics (NAMD). See SI Appendix for details.

Stimuli-Responsive Studies of Nanotube Formation. For a demonstration of stacking reversibility (Movie S7) and light-induced nanotube formation (Movie S8), see SI Appendix.

1. lijima S (1991) Helical microtubules of graphitic carbon. Nature 354:56-58.

2. Tans SJ, et al. (1997) Individual single-wall carbon nanotubes as quantum wires. Nature 386:474-477.

3. Percec V, et al. (2004) Self-assembly of amphiphilic dendritic dipeptides into helical pores. Nature 430:764-768.

4. Lee CY, Choi W, Han J-H, Strano MS (2010) Coherence resonance in a single-walled carbon nanotube ion channel. Science 329:1320-1324.

5. Langford GM (1995) Actin- and microtubule-dependent organelle motors: Interrelationships between the two motility systems. Curr Opin Cell Biol 7:82-88.

6. Tunuguntla RH, et al. (2017) Enhanced water permeability and tunable ion selectivity in subnanometer carbon nanotube porins. Science 357:792-796.

7. Keber FC, et al. (2014) Topology and dynamics of active nematic vesicles. Science 345 : 1135-1139.

8. Marvin DA (1998) Filamentous phage structure, infection and assembly. Curr Opin Struct Biol 8:150-158.

9. Hill JP, et al. (2004) Self-assembled hexa-peri-hexabenzocoronene graphitic nanotube. Science 304:1481-1483.

10. Percec V, Schlueter D, Ungar G, Cheng SZD, Zhang A (1998) Hierarchical control of internal superstructure, diameter, and stability of supramolecular and macromolecular column generated from tapered monodendritic building blocks. Macromolecules 31:1745-1762.

11. Gloe K, ed (2005) Macrocyclic Chemistry: Current Trends and Future Perspectives (Springer, Dordrecht, The Netherlands).

12. Bong DT, Clark TD, Granja JR, Ghadiri MR (2001) Self-assembling organic nanotubes. Angew Chem Int Ed Engl 40:988-1011.

13. Ghadiri MR, Granja JR, Milligan RA, McRee DE, Khazanovich N (1993) Self-assembling organic nanotubes based on a cyclic peptide architecture. Nature 366:324-327.

14. Fukino T, et al. (2014) Manipulation of discrete nanostructures by selective modulation of noncovalent forces. Science 344:499-504.

15. Rubin DJ, et al. (2015) Structural, nanomechanical, and computational characterization of D,L-cyclic peptide assemblies. ACS Nano 9:3360-3368.

16. Sessler JL, et al. (2003) The first liquid-crystalline, expanded porphyrins. Chem Commun 2422-2423.

17. Kawano S, Inada H, Tanaka K (2016) Self-assembled synthesis and liquid crystalline property of metallomacrocycle composed of naphthalenedihydroxamate and $\mathrm{Cu}^{2+}$ ions. Chem Lett 45:1105-1107.

18. Kawano S, Ishida Y, Tanaka K (2015) Columnar liquid-crystalline metallomacrocycles. J Am Chem Soc 137:2295-2302.

19. Sessler JL, et al. (2006) Schiff base oligopyrrolic macrocycles as ligands for lanthanides and actinides. J Alloys Compd 418:171-177.

20. Stepień M, Donnio B, Sessler JL (2007) Supramolecular liquid crystals based on cyclo[8]pyrrole. Angew Chem Int Ed Engl 46:1431-1435.

21. Kuritani M, Tashiro S, Shionoya M (2013) Organic and organometallic nanofiber formed by supramolecular assembly of diamond-shaped macrocyclic ligands and $\mathrm{Pd}^{(11)}$ complexes. Chem Asian J 8:1368-1371.

22. Lydon J (2010) Chromonic review. J Mater Chem 20:10071-10099.

23. Kumar RK, Yu X, Patil AJ, Li M, Mann S (2011) Cytoskeletal-like supramolecular assembly and nanoparticle-based motors in a model protocell. Angew Chem Int Ed Engl 50:9343-9347.

24. Ge P, et al. (2015) Atomic structures of a bactericidal contractile nanotube in its preand postcontraction states. Nat Struct Mol Biol 22:377-382.

25. Mann S (2009) Self-assembly and transformation of hybrid nano-objects and nanostructures under equilibrium and non-equilibrium conditions. Nat Mater 8:781-792.

26. Tseng RJ, et al. (2006) Digital memory device based on tobacco mosaic virus conjugated with nanoparticles. Nat Nanotechnol 1:72-77.

27. Chen X, et al. (2010) Virus-enabled silicon anode for lithium-ion batteries. ACS Nano 4:5366-5372.

28. Niu Z, et al. (2007) Biological templated synthesis of water-soluble conductive polymeric nanowires. Nano Lett 7:3729-3733.
Thiol-Ene Cross-Linking of Nanotubes and XPS Analyses. Photo-cross-linking of nanotubes deposited on HOPG and in solution was conducted using a setup illustrated in SI Appendix.

ACKNOWLEDGMENTS. We thank Yimo Han and Dr. Reiner Bleher for advice on electron microscopy; Leilei Xiao for assistance in mass spectrometry Dr. Sumit Kewalramani, Dr. Michio Matsumoto, Dr. Amanda Corcos for assistance in X-ray diffraction analyses; and Chong Luo and Prof. Jiaxing Huang for assistance with photography using cross-polarizers. C.S. acknowledges the support of the Cornell Materials Research Science and Engineering Center (MRSEC) program (NSF DMR-1719875). W.R.D. and A.D.C. acknowledge the US Army Research Office for Grant W911NF-15-1-0447. A.M.E. is supported by the National Science Foundation (NSF) Graduate Research Fellowship (DGE-1324585) and the Ryan Fellowship from the Northwestern University. N.C.F. was supported by the US Department of Energy (DOE), through Contract DE-AC02-06CH11357. M.S. B.H., M.O.d.I.C., and M.J.B. are supported by US DOE Grant DE-FG02-08ER46539. M.O.d.I.C. thanks The Sherman Fairchild Foundation for computational support. X.L. and M.C.H. acknowledge the Northwestern University MRSEC (NSF DMR-1720139) for support of the AFM characterization. A.D.C. was supported through a National Defense Science and Engineering Graduate Fellowship. Finally, we acknowledge the Biomedical Image Group at École Polytechnique Fédérale de Lausanne (EPFL), Switzerland for use of OrentationJ.

29. Aida T, Meijer EW, Stupp SI (2012) Functional supramolecular polymers. Science 335 813-817.

30. Song W, Kinloch IA, Windle AH (2003) Nematic liquid crystallinity of multiwall carbon nanotubes. Science 302:1363.

31. Zhang J, Moore JS (1994) Nanoarchitectures. 6. Liquid crystals based on shapepersistent macrocyclic mesogens. J Am Chem Soc 116:2655-2656.

32. Chavez AD, et al. (2018) Equilibration of imine-linked polymers to hexagonal macrocycles driven by self-assembly. Chemistry 24:3989-3993.

33. Favrot J, Vocelle D, Sandorfy C (1979) Infrared and Raman studies on some imines and their picrates. Relation to the problems of protonation in visual pigments. Photochem Photobiol 30:417-421.

34. Wang $Y$, Poirier RA (1997) Factors that influence the CN stretching frequency in imines. J Phys Chem A 101:907-912.

35. Onsager $L$ (1949) The effects of shape on the interaction of colloidal particles. Ann $N$ $Y$ Acad Sci 51:627-659.

36. Sing CE, Zwanikken JW, Olvera de la Cruz M (2014) Electrostatic control of block copolymer morphology. Nat Mater 13:694-698.

37. Kwon H-K, Pryamitsyn VA, Zwanikken JW, Shull KR, Olvera de la Cruz M (2017) Solubility and interfacial segregation of salts in ternary polyelectrolyte blends. Soft Matter 13:4830-4840

38. Brown JR, Seo Y, Hall LM (2018) Ion correlation effects in salt-doped block copolymers. Phys Rev Lett 120:127801.

39. Torrie GM, Valleau JP (1977) Nonphysical sampling distributions in Monte Carlo freeenergy estimation: Umbrella sampling. J Comput Phys 23:187-199.

40. Chu J-W, Voth GA (2007) Coarse-grained free energy functions for studying protein conformational changes: A double-well network model. Biophys J 93:3860-3871.

41. Hur S-M, et al. (2015) Molecular pathways for defect annihilation in directed selfassembly. Proc Natl Acad Sci USA 112:14144-14149.

42. Solis FJ, de la Cruz MO (2000) Collapse of flexible polyelectrolytes in multivalent salt solutions. J Chem Phys 112:2030-2035.

43. Sept D, McCammon JA (2001) Thermodynamics and kinetics of actin filament nucleation. Biophys J 81:667-674.

44. Jiménez-Ángeles F, Messina R, Holm C, Lozada-Cassou M (2003) lon pairing in mode electrolytes: A study via three-particle correlation functions. J Chem Phys 119 4842-4856.

45. Hall LM, Stevens MJ, Frischknecht AL (2011) Effect of polymer architecture and ionic aggregation on the scattering peak in model ionomers. Phys Rev Lett 106:127801.

46. Vögele M, Holm C, Smiatek J (2015) Coarse-grained simulations of polyelectrolyte complexes: MARTINI models for poly(styrene sulfonate) and poly(diallyldimethylammonium). J Chem Phys 143:243151.

47. Ma B, Nguyen TD, Pryamitsyn VA, Olvera de la Cruz M (2018) lonic correlations in random ionomers. ACS Nano 12:2311-2318.

48. Hobza P, Müller-Dethlefs K (2009) Non-Covalent Interactions (R Soc Chem, Cambridge, UK)

49. Moonoosawmy KR, Kruse P (2008) To dope or not to dope: The effect of sonicating single-wall carbon nanotubes in common laboratory solvents on their electronic structure. J Am Chem Soc 130:13417-13424.

50. Castner DG, Hinds K, Grainger DW (1996) X-ray photoelectron spectroscopy sulfur $2 p$ study of organic thiol and disulfide binding interactions with gold surfaces. Langmuir 12:5083-5086.

51. Zhang R, Kumar N, Ross JL, Gardel ML, de Pablo JJ (2018) Interplay of structure, elasticity, and dynamics in actin-based nematic materials. Proc Natl Acad Sci USA 115 E124-E133.

52. Ohkubo K, Mase K, Karnas E, Sessler JL, Fukuzumi S (2014) Cyclo[8]pyrrole: An androgynous expanded porphyrin that acts as both an electron donor and acceptor in anion-bound supramolecular electron donor-acceptor complexes.J Phys Chem C 118: 18436-18444.

53. Wu R-T, Chi X, Hirao T, Lynch VM, Sessler JL (2018) Supramolecular properties of a monocarboxylic acid-functionalized "Texas-sized" molecular box. J Am Chem Soc 140:6823-6831. 